\title{
The Crystal Structure of $\left[\left(\mathrm{NH}_{3}\right)_{4} \mathrm{Co}(\mathrm{OH})_{2} \mathrm{Co}\left(\mathrm{NH}_{3}\right)_{4}\right] \mathrm{Cl}_{4} \cdot 4 \mathrm{H}_{2} \mathrm{O}$
}

\author{
NILS-GÖSTA VANNERBERG
}

Department of Inorganic Chemistry, Chalmers University of Technology, Göteborg, Sweden

\begin{abstract}
In a systematic study of the structures of dinuclear cobalt complexes the crystal structure of $\left[\left(\mathrm{NH}_{3}\right)_{4} \mathrm{Co}(\mathrm{OH})_{2} \mathrm{Co}\left(\mathrm{NH}_{3}\right)_{4}\right] \mathrm{Cl}_{4} \cdot 4 \mathrm{H}_{2} \mathrm{O}$ has been determined. The elementary cell is triclinic with $a=6.66 \AA$, $b=7.82 \AA, c=10.04 \AA, \alpha=92^{\circ} 2, \beta=106^{\circ} 8$ and $\gamma=106^{\circ} 6$. The coordination about each cobalt ion is nearly octahedral, the two octahedrons having one edge in common.
\end{abstract}

Octaammonia- $\mu$-dihydroxodicobalt(III)tetrachloride-4-water

$\left[\left(\mathrm{NH}_{3}\right)_{4} \mathrm{Co}_{\mathrm{OH}}^{\mathrm{OH}} \mathrm{Co}\left(\mathrm{NH}_{3}\right)_{4}\right] \mathrm{Cl}_{4} \cdot 4 \mathrm{H}_{2} \mathrm{O}$ was first prepared by Gentele in

$1856^{1}$. Werner reinvestigated this and other compounds containing the same complex cation in $1907^{2-5}$. He also proposed a geometrical configuration for the complex ion, which, as will be seen below, agrees very well with that described in this paper. Dubsky ${ }^{6}$ and Frank ${ }^{7}$, also, described the preparation of the compound and its chemical behaviour.

The compound is red and diamagnetic ${ }^{8-10}$. The electrical conductivity in diluted hydrochloric acid indicates a dinuclear cobalt complex with a formula weight ${ }^{14}$ around 300 . The light absorption in the visible and ultraviolet regions has been registered by several authors ${ }^{11-13}$. Absorption has been found ${ }^{12}$ at $100 \times 10^{13} \mathrm{sec}^{-1}$ and $130 \times 10^{13} \mathrm{sec}^{-1}$.

\section{EXPERIMENTAL}

The preparation method by Werner for $\left[\left(\mathrm{NH}_{3}\right)_{4} \mathrm{Co}(\mathrm{OH})_{2} \mathrm{Co}\left(\mathrm{NH}_{3}\right)_{4}\right] \mathrm{Cl}_{4} \cdot 4 \mathrm{H}_{2} \mathrm{O}$ was applied ${ }^{2}$. The synthesis contained several steps. First $\left[\mathrm{CoCO}_{3}\left(\mathrm{NH}_{3}\right)_{4}\right]_{2} \mathrm{SO}_{4} \cdot 3 \mathrm{H}_{2} \mathrm{O}$ was prepared from cobalt(II) carbonate, sulphuric acid, air, ammonia and ammonium carbonate in the mentioned order.

$\mathrm{CoCO}_{3}(\mathrm{~s})+2 \mathrm{H}+\rightarrow \mathrm{Co}^{2}++\mathrm{H}_{2} \mathrm{O}+\mathrm{CO}_{2}(\mathrm{~g})$

$4 \mathrm{Co}^{2+}+\mathrm{O}_{2}+4 \mathrm{H}++12 \mathrm{SO}_{4}^{2-} \rightarrow 4\left[\mathrm{Co}\left(\mathrm{SO}_{4}\right)_{3}\right]^{3-}+2 \mathrm{H}_{2} \mathrm{O}$

$2\left[\mathrm{Co}\left(\mathrm{SO}_{4}\right)_{3}\right]^{2-}+2 \mathrm{CO}_{3}{ }^{2-} \rightarrow 8 \mathrm{NH}_{3}+3 \mathrm{H}_{2} \mathrm{O} \rightarrow 2\left[\mathrm{CoCO}_{3}\left(\mathrm{NH}_{3}\right)_{4}\right] \mathrm{SO}_{4}$.

$3 \mathrm{H}_{2} \mathrm{O}(\mathrm{s})+5 \mathrm{SO}_{4}{ }^{2-}$

Acta Chem. Scand. 17 (1963) No. 1 
$\left[\mathrm{CoCO}_{3}\left(\mathrm{NH}_{3}\right)_{4}\right]_{2} \mathrm{SO}_{4} \cdot 3 \mathrm{H}_{2} \mathrm{O}$ was then treated by diluted sulphuric acid and alcohol, whereupon $\left[\mathrm{Co}\left(\mathrm{NH}_{3}\right)_{4}\left(\mathrm{H}_{2} \mathrm{O}\right)_{2}\right]_{2}\left(\mathrm{SO}_{4}\right)_{3} \cdot 3 \mathrm{H}_{2} \mathrm{O}$ was formed.

$\left[\mathrm{CoCO}_{3}\left(\mathrm{NH}_{3}\right)_{4}\right]_{2} \mathrm{SO}_{4} \cdot 3 \mathrm{H}_{2} \mathrm{O}(\mathrm{s})+2 \mathrm{SO}_{4}{ }^{2-}+4 \mathrm{H}++4 \mathrm{H}_{2} \mathrm{O}+\rightarrow\left[\mathrm{Co}\left(\mathrm{H}_{2} \mathrm{O}\right)_{2}\left(\mathrm{NH}_{3}\right)_{4}\right]_{2}\left[\mathrm{SO}_{4}\right)_{3}$.

$3 \mathrm{H}_{2} \mathrm{O}(\mathrm{s})$.

$\left[\mathrm{Co}\left(\mathrm{H}_{2} \mathrm{O}\right)_{2}\left(\mathrm{NH}_{3}\right)_{4}\right]_{2}\left(\mathrm{SO}_{4}\right)_{3} \cdot 3 \mathrm{H}_{2} \mathrm{O}$ was treated with diluted ammonia and alcohol until the hydroxy sulphate of the same complex cation was formed. If this salt is heated to $100^{\circ}$ it releases water and a dinuclear cobalt $\mu$-oxo complex is formed. At lower tempera. tures the complex reacts again with water and $\left[\mathrm{Co}(\mathrm{OH})\left(\mathrm{H}_{2} \mathrm{O}\right)\left(\mathrm{NH}_{3}\right)_{4}\right] \mathrm{SO}_{4} \cdot \mathrm{H}_{2} \mathrm{O}$ is formed. $\left[\mathrm{Co}\left(\mathrm{H}_{2} \mathrm{O}\right)_{2}\left(\mathrm{NH}_{3}\right)_{4}\right]_{2}\left(\mathrm{SO}_{4}\right)_{3} \cdot 3 \mathrm{H}_{2} \mathrm{O}(\mathrm{s})+\mathrm{OH}^{-} \rightarrow\left[\mathrm{Co}\left(\mathrm{H}_{2} \mathrm{O}\right)_{2}\left(\mathrm{NH}_{3}\right)_{4}\right] \mathrm{SO}_{4} \mathrm{OH}(\mathrm{s})$

$2\left[\mathrm{Co}\left(\mathrm{H}_{2} \mathrm{O}\right)_{2}\left(\mathrm{NH}_{3}\right)_{4}\right] \mathrm{SO}_{4} \mathrm{OH} \stackrel{100^{\circ}}{\longrightarrow}\left[\mathrm{H}_{2} \mathrm{O}\left(\mathrm{NH}_{3}\right)_{4} \mathrm{CoOCo}\left(\mathrm{NH}_{3}\right)_{4} \mathrm{H}_{2} \mathrm{O}\right]_{2}\left(\mathrm{SO}_{4}\right)_{2}(\mathrm{~s})+2 \mathrm{H}_{2} \mathrm{O}(\mathrm{g})$

$\left[\mathrm{H}_{2} \mathrm{O}\left(\mathrm{NH}_{3}\right)_{4} \mathrm{CoOCo}\left(\mathrm{NH}_{3}\right)_{4} \cdot \mathrm{H}_{2} \mathrm{O}\right]\left(\mathrm{SO}_{4}\right)_{2}+3 \mathrm{H}_{2} \mathrm{O} \rightarrow 2\left[\mathrm{Co}(\mathrm{OH})\left(\mathrm{H}_{2} \mathrm{O}\right)\left(\mathrm{NH}_{3}\right)_{4}\right] \mathrm{SO}_{4} \cdot \mathrm{H}_{2} \mathrm{O}$.

When the last mentioned salt was again heated to $100^{\circ} \mathrm{C}$, water was lost and $\left[\left(\mathrm{NH}_{3}\right)_{4} \mathrm{Co}(\mathrm{OH})_{2} \mathrm{Co}\left(\mathrm{NH}_{3}\right)_{4}\right]\left(\mathrm{SO}_{4}\right)_{2}$ was formed. The final product,

$\left[\left(\mathrm{NH}_{3}\right)_{4} \mathrm{Co}(\mathrm{OH})_{2} \mathrm{Co}\left(\mathrm{NH}_{3}\right)_{4} \mathrm{Cl}_{4}\left(\mathrm{H}_{2} \mathrm{O}\right)_{4}\right.$, was obtained, when the sulphate was treated by a water solution of ammonium chloride.

$\left[\left(\mathrm{NH}_{3}\right)_{4} \mathrm{Co}(\mathrm{OH})_{2} \mathrm{Co}\left(\mathrm{NH}_{3}\right)_{4}\right]\left(\mathrm{SO}_{4}\right)_{2}(\mathrm{~s})+4 \mathrm{Cl}^{-}+4 \mathrm{H}_{2} \mathrm{O} \rightarrow$

$\left[\left(\mathrm{NH}_{3}\right)_{4} \mathrm{Co}\left(\mathrm{OH}_{2} \mathrm{Co}\left(\mathrm{NH}_{3}\right)_{4}\right] \mathrm{Cl}_{4} \cdot 4 \mathrm{H}_{2} \mathrm{O}(\mathrm{s})\right.$.

Analysis. Found: $\mathrm{Co} 23.1 ; \mathrm{NH}_{3} 25.5 ; \mathrm{Cl} 28.2$. Calc. $\mathrm{Co} 23.5 ; \mathrm{NH}_{3} 27.1 ; \mathrm{Cl} 28.3$.

\section{DETERMINATION OF CRYSTAL STRUCTURE}

The elementary cell dimensions were obtained from single crystal photographs using rotation and Weissenberg methods. The cell was found to be triclinic

$$
\begin{array}{ll}
a=6.66 \pm 0.02 & \alpha=92.2 \pm 0.2 \\
b=7.82 \pm 0.02 & \beta=106.8 \pm 0.2 \\
c=10.04 \pm 0.03 & \gamma=106.6 \pm 0.2
\end{array}
$$

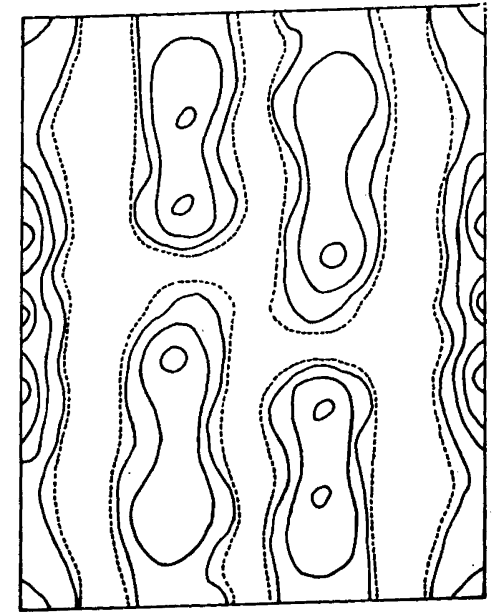

Fig. 1. Patterson projection on (100).

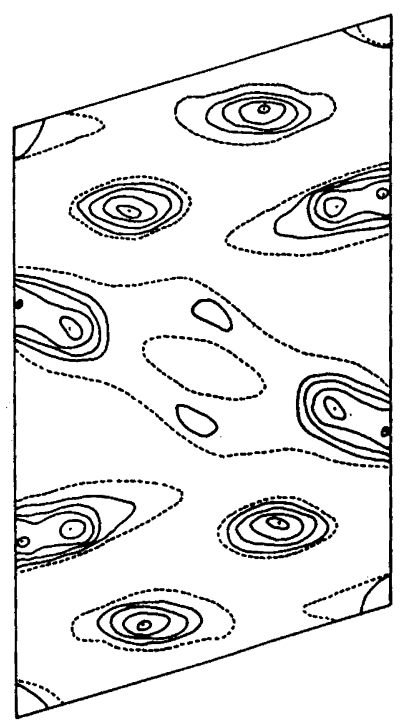

Fig. 2. Pattersonprojection on (010).

Acta Chem. Scand. 17 (1963) No. 1 
Table 1. Atomic coordinates for $\left[\left(\mathrm{NH}_{3}\right)_{4} \mathrm{Co}(\mathrm{OH})_{2} \mathrm{Co}\left(\mathrm{NH}_{3}\right)_{4}\right] \mathrm{Cl}_{4} \cdot 4 \mathrm{H}_{2} \mathrm{O}$.

$\begin{array}{lccc} & x & y & z \\ \mathrm{Co} & 0.012 & 0.005 & 0.150 \\ \mathrm{Cl}_{1} & 0.159 & 0.308 & 0.537 \\ \mathrm{Cl}_{2} & 0.356 & 0.662 & 0.053 \\ \mathrm{~N}_{1}\left(\mathrm{NH}_{3}\right) & 0.065 & 0.273 & 0.159 \\ \mathrm{~N}_{2}\left(\mathrm{NH}_{3}\right) & 0.969 & 0.753 & 0.151 \\ \mathrm{~N}_{3}\left(\mathrm{NH}_{3}\right) & 0.235 & 0.023 & 0.319 \\ \mathrm{~N}_{4}\left(\mathrm{NH}_{3}\right) & 0.803 & 0.998 & 0.252 \\ \mathrm{O}_{1}(\mathrm{OH}) & 0.200 & 0.020 & 0.026 \\ \mathrm{O}_{2}\left(\mathrm{H}_{2} \mathrm{O}\right) & 0.337 & 0.708 & 0.416 \\ \mathrm{O}_{3}\left(\mathrm{H}_{2} \mathrm{O}\right) & 0.376 & 0.676 & 0.752\end{array}$

The observed density was $1.74 \mathrm{~g} / \mathrm{cm}^{3}$, the calculated density assuming one formula unit in the elementary cell $1.76 \mathrm{~g} / \mathrm{cm}^{3}$. The space group was assumed to be $P \overline{1}$.

The intensities of the $\mathrm{X}$-ray reflexions were determined by Weissenberg methods using multiple film technique. Most zones detectable with $\mathrm{Fe} K \alpha$ radiation were registered.

Patterson projections along the two shortest axes were first performed and probable positions for the cobalt and chloride atoms were determined by a superposition method. The heaviest peaks were identified as $\mathrm{Co}-\mathrm{Cl}$ peaks, the second heaviest as Co-Co peaks; see Figs. 1 and 2.

Knowing the positions of the cobalt and chloride ions, the signs of most structure factors could be determined. Electron density projections on (100) and $(010)$ gave the approximate positions of most atoms. The accuracy of the atom positions was low due to considerable atomic overlap in all directions. The structure was refined by repeated difference syntheses using the $h 0 l$ and $0 \mathrm{kl}$ reflexions only. When no further improvement in the agreement between observed and calculated structure factors could be attained, the signs of all structure factors were calculated, using the atomic parameter values obtained from the difference synthesis. A complete three-dimensional Fourier synthesis was then performed with the aid of a Besk electronic digital computer, using programs devised by Westman et al. ${ }^{15}$ and by Åsbrink et al. ${ }^{16}$ A comparison between observed and calculated structure factors can be found in Table 2, the atomic parameters in Table 1. The reliability index $R=\Sigma|| F_{\mathrm{o}}|-| F_{\mathrm{c}} \||\Sigma| F_{\mathrm{o}} \mid$ was calculated to be 0.19 . An isotropic temperature factor with $B=1.30 \AA^{2}$ was applied. The standard deviations of the atomic parameter were calculated by Cruickshank's method ${ }^{17}$, but to cover the variation in some bond lengths these figures were multiplied with two.

$$
\begin{gathered}
\text { Description of the structure of } \\
{\left[\left(\mathrm{NH}_{3}\right)_{4} \mathrm{Co}(\mathrm{OH})_{2} \mathrm{Co}\left(\mathrm{NH}_{3}\right)_{4}\right] \mathrm{Cl}_{4} \cdot 4 \mathrm{H}_{2} \mathrm{O}}
\end{gathered}
$$

The crystal structure is built up of the complex ion $\left[\left(\mathrm{NH}_{3}\right)_{4} \mathrm{Co}(\mathrm{OH})_{2} \mathrm{Co}\left(\mathrm{NH}_{3}\right)_{4}\right]^{4+}$, chloride ions and water molecules. The hydrogen bonds between the three species are weak to moderately strong. 
Table 2.

\begin{tabular}{|c|c|c|c|c|c|c|c|c|}
\hline$h k l$ & $F_{\mathrm{o}}$ & $F_{\mathrm{c}}$ & $h k l$ & $F_{\mathrm{o}}$ & $F_{\mathrm{c}}$ & $h k l$ & $F_{\mathrm{o}}$ & $F_{\mathrm{c}}$ \\
\hline 001 & 16.3 & 20.7 & $\mathbf{l}$ & - & 0.1 & $1 \overline{1} 0$ & 26.1 & 30.8 \\
\hline 2 & 5.3 & 6.5 & 2 & -11.0 & -14.3 & 1 & 4.7 & 4.7 \\
\hline $\mathbf{3}$ & -29.2 & -23.3 & 3 & -9.8 & -15.7 & 2 & 4.3 & 4.3 \\
\hline 4 & - & -1.7 & 4 & -8.1 & -6.9 & 3 & 25.6 & -18.4 \\
\hline 5 & - & -0.1 & $40 \overline{1}$ & 18.6 & 19.0 & 4 & 4.6 & -2.7 \\
\hline 6 & 12.6 & 9.5 & $\overline{2}$ & -11.6 & -12.7 & 5 & - & -1.6 \\
\hline 7 & 11.0 & 16.2 & $\underline{\overline{3}}$ & - & -4.4 & 6 & 30.0 & 30.0 \\
\hline 8 & - & 1.9 & $\underline{4}$ & -21.4 & -27.8 & 7 & 4.8 & 7.6 \\
\hline 9 & 6.4 & 10.5 & $\overline{5}$ & - & 5.5 & 8 & 5.3 & 9.7 \\
\hline 100 & 21.8 & 31.7 & $\overline{6}$ & 9.2 & 12.6 & 1 & 10.4 & 12.3 \\
\hline 1 & 4.0 & 6.1 & 500 & 13.7 & 20.6 & $\underline{\overline{2}}$ & 14.4 & -15.0 \\
\hline 2 & -15.9 & -15.4 & 1 & - & 3.4 & $\underline{\overline{3}}$ & 31.7 & -33.8 \\
\hline 3 & -39.5 & -48.8 & 2 & - & -1.2 & $\underline{\overline{4}}$ & 15.1 & -17.5 \\
\hline 4 & -35.6 & -33.2 & $\underline{3}$ & - & -3.3 & $\underline{\overline{5}}$ & 3.4 & -5.6 \\
\hline 5 & - & 2.2 & $50 \overline{1}$ & 12.9 & 14.5 & $\overline{\mathbf{6}}$ & - & -4.0 \\
\hline 6 & - & 9.8 & $\overline{2}$ & - & -1.2 & $\overline{7}$ & 17.5 & 17.9 \\
\hline 7 & 12.0 & 12.1 & $\overline{3}$ & - & -13.7 & & & \\
\hline 8 & - & -6.1 & 600 & 13.1 & 18.3 & 210 & 28.0 & -23.0 \\
\hline $10 \overline{\overline{1}}$ & 12.6 & 11.4 & 1 & - & 0.5 & 1 & 2.3 & 5.5 \\
\hline$\overline{2}$ & - & -0.3 & $60 \overline{1}$ & - & 5.7 & 2 & 11.0 & -11.6 \\
\hline$\overline{\mathbf{3}}$ & -19.7 & -20.0 & 010 & 19.9 & 25.3 & 3 & 33.2 & -30.5 \\
\hline$\overline{4}$ & - & -1.3 & 1 & 13.4 & 21.0 & 4 & 15.8 & -17.5 \\
\hline$\overline{\mathbf{5}}$ & -9.8 & -7.3 & 2 & 22.2 & -22.2 & 5 & - & -6.0 \\
\hline$\overline{6}$ & 37.1 & 38.8 & 3 & 25.6 & 17.2 & 6 & 5.0 & 9.5 \\
\hline$\overline{7}$ & 26.2 & 26.6 & 4 & 13.1 & -14.6 & 7 & - & -1.2 \\
\hline$\overline{8}$ & 16.0 & 19.3 & 5 & 16.0 & 13.4 & 8 & - & 4.8 \\
\hline 200 & 4.8 & 4.5 & 6 & 14.1 & 12.7 & $\overline{\mathbf{1}}$ & 6.6 & 13.2 \\
\hline 1 & 25.9 & 18.3 & 7 & 21.1 & 23.1 & $\overline{2}$ & 4.0 & -11.9 \\
\hline 2 & -26.2 & -19.4 & 8 & 5.2 & 5.0 & $\overline{\mathbf{3}}$ & 5.3 & 1.9 \\
\hline 3 & -9.3 & -8.3 & $\overline{1}$ & 17.3 & 15.0 & $\overline{4}$ & 21.8 & 25.5 \\
\hline 4 & -23.3 & -22.5 & $\overline{\overline{2}}$ & 15.5 & -14.4 & $\overline{\overline{5}}$ & 6.3 & 9.1 \\
\hline 5 & 15.5 & 16.7 & $\overline{3}$ & 44.4 & -37.5 & 6 & 10.2 & 13.3 \\
\hline 6 & 8.2 & 13.0 & $\overline{4}$ & 12.5 & -8.9 & $\overline{7}$ & 18.8 & 20.4 \\
\hline 7 & 21.2 & 18.3 & $\overline{5}$ & 13.0 & -13.9 & $\overline{8}$ & 5.2 & 8.0 \\
\hline 8 & - & 1.7 & $\overline{6}$ & 18.1 & 17.3 & $2 \overline{10}$ & 34.6 & 49.2 \\
\hline $20 \overline{\mathrm{I}}$ & 20.0 & 18.3 & $\overline{7}$ & 5.9 & 5.0 & 1 & 12.6 & 7.0 \\
\hline$\overline{2}$ & -6.1 & -8.0 & $\overline{8}$ & 9.2 & 7.6 & 2 & 2.2 & 4.6 \\
\hline$\overline{\mathbf{3}}$ & -54.1 & -54.0 & $\overline{9}$ & 4.9 & -4.4 & 3 & 38.1 & 34.7 \\
\hline$\overline{4}$ & -31.2 & -26.9 & & & & 4 & 14.4 & 14.9 \\
\hline$\overline{\mathbf{5}}$ & -20.2 & -17.4 & 110 & 34.5 & 35.3 & 5 & - & 2.2 \\
\hline$\overline{6}$ & 20.0 & 17.1 & 1 & 35.5 & 31.1 & 6 & 4.8 & 2.5 \\
\hline$\overline{7}$ & - & 5.5 & 2 & 6.4 & -9.5 & 7 & 3.4 & 2.8 \\
\hline$\overline{8}$ & 17.5 & 11.1 & 3 & 3.7 & -7.4 & 8 & - & 5.0 \\
\hline 300 & 25.0 & 20.4 & 4 & 15.6 & -16.6 & $\underline{1}$ & 3.6 & 6.3 \\
\hline 1 & 29.2 & 21.9 & 5 & 5.1 & 8.4 & $\overline{2}$ & 15.9 & 19.5 \\
\hline 2 & -16.5 & -16.0 & 6 & 3.1 & -4.3 & $\overline{\mathbf{3}}$ & 21.3 & 23.9 \\
\hline 3 & -7.0 & -14.0 & 7 & - & 0.9 & $\overline{4}$ & 8.4 & 5.7 \\
\hline 4 & -15.6 & -16.5 & 8 & - & -2.7 & $\underline{5}$ & - & 2.0 \\
\hline 5 & - & 6.3 & $\overline{1}$ & 33.5 & 35.6 & $\underline{\overline{6}}$ & 5.4 & 6.8 \\
\hline 6 & - & 1.8 & $\underline{2}$ & 5.5 & 9.9 & $\overline{7}$ & 10.7 & 15.6 \\
\hline $30 \overline{1}$ & 27.2 & 25.3 & $\underline{\overline{\mathbf{3}}}$ & 11.9 & 8.9 & $\overline{8}$ & - & 0.9 \\
\hline$\overline{\mathbf{2}}$ & - & -0.4 & $\underline{\overline{4}}$ & 3.0 & 5.3 & $\overline{9}$ & 4.7 & 8.1 \\
\hline$\overline{\mathbf{3}}$ & -7.4 & -7.3 & $\overline{\mathbf{5}}$ & 3.6 & 2.2 & & & \\
\hline$\overline{4}$ & -27.3 & -27.2 & $\underline{\mathbf{6}}$ & - & 1.6 & 310 & 3.7 & 5.9 \\
\hline$\overline{\mathbf{5}}$ & - & 1.9 & $\overline{7}$ & 11.2 & 14.5 & 1 & - & 0.6 \\
\hline$\overline{6}$ & 7.0 & 6.2 & $\overline{8}$ & 5.0 & 6.2 & 2 & - & 0.6 \\
\hline 400 & - & 2.0 & $\overline{9}$ & 11.7 & 14.1 & 3 & 2.8 & -4.7 \\
\hline
\end{tabular}


DINUCLEAR COBALT COM PLEXES

\begin{tabular}{|c|c|c|c|c|c|c|c|c|}
\hline$h k l$ & $F_{\mathrm{o}}$ & $F_{\mathrm{c}}$ & $h k l$ & $F_{\mathrm{o}}$ & $F_{\mathrm{c}}$ & $h k l$ & $F_{\mathrm{o}}$ & $F_{\mathrm{c}}$ \\
\hline 4 & 3.8 & 4.5 & $\overline{\mathbf{6}}$ & 9.0 & 12.5 & 5 & - & 4.1 \\
\hline$\underline{5}$ & - & -1.7 & $\overline{7}$ & 7.3 & 7.3 & 6 & - & -2.0 \\
\hline$\underline{\underline{I}}$ & - & 2.8 & & & & 7 & - & -3.1 \\
\hline$\overline{2}$ & - & -6.0 & $5 \overline{1} 0$ & 18.7 & 19.3 & 8 & - & 0.8 \\
\hline$\overline{\mathbf{3}}$ & 23.8 & 25.5 & 1 & 13.1 & 12.0 & $\underline{9}$ & 8.3 & 7.1 \\
\hline$\underline{\underline{4}}$ & 24.4 & 27.2 & $\underline{2}$ & 7.7 & -11.2 & $\overline{\mathbf{I}}$ & 24.6 & 29.2 \\
\hline$\overline{5}$ & 4.0 & 2.9 & $\underline{I}$ & 23.0 & 22.2 & $\underline{\overline{2}}$ & 5.9 & -5.5 \\
\hline$\underline{6}$ & 9.3 & 6.4 & $\underline{\overline{2}}$ & - & 0.6 & $\underline{\overline{\mathbf{3}}}$ & 16.8 & 13.8 \\
\hline \multirow[t]{2}{*}{$\overline{7}$} & 5.7 & 4.0 & $\overline{\overline{3}}$ & - & 3.0 & $\overline{4}$ & - & -0.2 \\
\hline & & & $\overline{\underline{4}}$ & - & -6.5 & $\underline{\overline{5}}$ & - & 5.0 \\
\hline $3 \overline{1} 0$ & 8.3 & 9.3 & $\overline{5}$ & - & 0.3 & $\overline{\underline{6}}$ & - & 2.5 \\
\hline 1 & 5.8 & -4.0 & $\underline{\underline{6}}$ & - & 5.5 & $\overline{7}$ & 10.1 & 11.5 \\
\hline 2 & 14.6 & -12.9 & $\overline{7}$ & 12.0 & 15.2 & $\underline{8}$ & - & 0.7 \\
\hline 3 & 24.3 & -28.2 & & & & $\overline{9}$ & - & -9.0 \\
\hline 4 & 15.7 & -19.2 & $6 \overline{1} \underline{0}$ & - & 0.6 & & & \\
\hline 5 & - & 1.6 & $\overline{\mathbf{I}}$ & 10.0 & 12.2 & 220 & 27.8 & 25.6 \\
\hline$\underline{6}$ & - & 9.0 & & & & 1 & 4.8 & 2.0 \\
\hline$\overline{1}$ & 2.2 & 1.5 & 020 & & & 2 & - & 8.0 \\
\hline$\underline{\overrightarrow{2}}$ & 2.4 & 4.3 & 1 & 13.4 & 15.5 & 3 & 24.1 & 22.6 \\
\hline$\overline{\mathbf{3}}$ & 31.4 & 28.8 & 2 & 13.2 & -16.0 & 4 & 3.9 & -2.5 \\
\hline$\underline{4}$ & 18.6 & 21.6 & 3 & 21.2 & -16.7 & 5 & 3.5 & -1.7 \\
\hline$\overline{\overline{5}}$ & 3.4 & 6.0 & 4 & 14.3 & -10.3 & 6 & 4.1 & 1.6 \\
\hline$\underline{6}$ & 27.3 & 27.5 & 5 & 10.7 & 9.9 & 7 & - & -0.9 \\
\hline$\overline{\overline{7}}$ & 8.4 & 8.4 & 6 & 8.3 & 9.1 & 1 & - & 0.5 \\
\hline \multirow[t]{2}{*}{$\overline{8}$} & 9.8 & 12.9 & 7 & - & 0.8 & $\overline{\underline{2}}$ & 12.2 & 11.2 \\
\hline & & & $\frac{1}{2}$ & 23.1 & 23.2 & $\overline{\mathbf{3}}$ & 3.3 & - 6.3 \\
\hline 410 & 17.4 & 26.9 & 2 & 18.1 & -17.5 & $\underline{4}$ & 8.0 & 8.2 \\
\hline 1 & - & 0.0 & $\underline{\mathbf{3}}$ & 24.2 & 22.1 & $\underline{5}$ & - & 0.2 \\
\hline 2 & - & 4.6 & $\overline{4}$ & 4.8 & -4.4 & $\overline{6}$ & - & 2.0 \\
\hline 3 & 6.8 & 9.7 & $\frac{5}{9}$ & 7.7 & 9.0 & $\frac{7}{9}$ & 6.8 & 9.0 \\
\hline 4 & - & 0.9 & 6 & 12.0 & 11.3 & 8 & - & 1.7 \\
\hline 5 & - & -3.7 & $\overline{7}$ & 17.2 & 17.6 & $2 \overline{2} 0$ & 9.5 & -16.1 \\
\hline$\overline{\mathbf{1}}$ & 11.0 & 4.7 & 8 & - & 6.4 & 1 & 6.7 & -4.7 \\
\hline$\overline{\underline{2}}$ & 11.9 & -9.4 & $\overline{9}$ & - & 0.5 & 2 & - & -2.4 \\
\hline$\overline{\mathbf{3}}$ & 一 & -0.9 & & & & 3 & 38.3 & -35.1 \\
\hline$\underline{\overline{4}}$ & 3.1 & -2.8 & 120 & 3.3 & 8.6 & 4 & 18.3 & -14.9 \\
\hline$\overline{\overline{5}}$ & 3.1 & -0.4 & 1 & - & -1.4 & 5 & 6.4 & -7.2 \\
\hline$\underline{\overline{6}}$ & - & 0.6 & 2 & - & 1.3 & 6 & 9.4 & 9.5 \\
\hline$\underline{\overline{7}}$ & 4.4 & 8.2 & 3 & 12.4 & -7.6 & 7 & - & 2.2 \\
\hline \multirow[t]{2}{*}{$\overline{8}$} & - & -3.2 & 4 & - & -1.3 & 8 & 4.1 & 3.6 \\
\hline & & & 5 & $\overline{17}$ & 0.0 & $\underline{1}$ & 5.0 & 5.8 \\
\hline 411 & 17.1 & 18.7 & 6 & 17.0 & 13.8 & $\overline{2}$ & 4.8 & -9.7 \\
\hline$\overline{2}$ & 8.9 & -7.1 & 7 & - & 2.4 & $\underline{\overline{3}}$ & 5.7 & 5.4 \\
\hline$\overline{\mathbf{3}}$ & 24.5 & -29.2 & 8 & 5.4 & 7.2 & $\overline{\underline{4}}$ & 17.3 & -20.9 \\
\hline$\overline{\underline{4}}$ & 13.3 & -17.7 & $\underline{1}$ & 4.9 & 3.9 & $\overline{\mathbf{5}}$ & 9.1 & 11.0 \\
\hline$\underline{5}$ & 14.6 & 15.6 & $\underline{\overline{2}}$ & 8.9 & -12.2 & $\underline{\overline{6}}$ & 7.2 & 7.5 \\
\hline$\underline{5}$ & - & 8.8 & $\underline{3}$ & 17.3 & -19.6 & 7 & 14.8 & 19.8 \\
\hline$\overline{6}$ & - & 2.0 & $\overline{4}$ & 12.7 & -14.3 & 8 & 2.9 & 4.8 \\
\hline \multirow[t]{2}{*}{$\overline{7}$} & 4.0 & 5.9 & $\overline{\mathbf{3}}$ & - & -0.4 & & & \\
\hline & & & 6 & 5.2 & -4.4 & 030 & 54.0 & 59.8 \\
\hline 510 & 8.6 & 6.2 & $\overline{7}$ & 15.4 & 14.2 & 1 & 8.5 & 7.1 \\
\hline 1 & 6.7 & -6.0 & $\overline{8}$ & - & -2.4 & 2 & 5.6 & 8.2 \\
\hline 2 & 7.2 & 3.5 & $\overline{8}$ & 5.9 & 4.4 & 3 & 19.8 & -16.8 \\
\hline$\overline{\mathbf{l}}$ & 3.5 & 2.0 & $1 \overline{2} 0$ & 27.4 & 30.6 & 4 & - & 5.0 \\
\hline$\overline{2}$ & - & 1.7 & 1 & 31.0 & 28.2 & 5 & - & 2.6 \\
\hline$\overline{\mathbf{3}}$ & 7.7 & 9.4 & 2 & 4.6 & 8.6 & 6 & 11.1 & 7.6 \\
\hline $\overrightarrow{4}$ & - & 2.0 & 3 & - & 0.6 & 7 & - & 5.5 \\
\hline$\overline{5}$ & 2.9 & -4.5 & 4 & 16.3 & 11.9 & 1 & 21.3 & 17.3 \\
\hline
\end{tabular}

Acta Chem. Scand. 17 (1963) No. 1 


\begin{tabular}{|c|c|c|c|c|c|c|c|c|}
\hline$h k l$ & $F_{\mathrm{o}}$ & $F_{\mathrm{c}}$ & $h k l$ & $F_{\mathrm{o}}$ & $F_{\mathrm{c}}$ & $h k l$ & $F_{\mathrm{o}}$ & $F_{\mathrm{c}}$ \\
\hline$\overline{2}$ & - & 0.8 & $2 \overline{3} 0$ & 10.1 & -8.2 & $\overline{\overline{2}}$ & 7.1 & 9.8 \\
\hline$\overline{\mathbf{3}}$ & 5.6 & 4.6 & 1 & 11.3 & 15.8 & $\overline{\mathbf{3}}$ & 19.9 & 22.8 \\
\hline$\underline{4}$ & - & 3.0 & 2 & 4.8 & 6.9 & $\underline{\overline{4}}$ & 6.5 & 11.1 \\
\hline$\overline{\overline{5}}$ & - & 0.7 & 3 & - & 0.7 & $\overline{\mathbf{5}}$ & - & 4.8 \\
\hline$\overline{6}$ & 5.6 & 0.4 & 4 & 11.8 & -13.9 & $\underline{\overline{6}}$ & 5.8 & 8.2 \\
\hline$\overline{7}$ & 8.6 & 11.3 & 5 & 4.6 & 8.4 & $\overline{7}$ & 10.5 & 8.6 \\
\hline \multirow[t]{2}{*}{$\overline{8}$} & - & 1.3 & 6 & 5.0 & 8.3 & & & \\
\hline & & & 7 & 8.9 & 8.6 & 240 & 7.7 & -7.3 \\
\hline 130 & 16.4 & 13.5 & $\overline{1}$ & 9.3 & 17.7 & 1 & 3.6 & 6.2 \\
\hline 1 & - & 0.8 & $\overline{\overline{2}}$ & 8.5 & -12.1 & 2 & 17.5 & -14.8 \\
\hline 2 & 8.7 & 9.1 & $\overline{\overline{3}}$ & 21.1 & -24.6 & 3 & 19.1 & -16.4 \\
\hline 3 & 30.2 & -29.0 & $\overline{\underline{4}}$ & 12.2 & -16.2 & 4 & 14.4 & -12.5 \\
\hline 4 & 15.0 & -16.0 & $\overline{\overline{5}}$ & 6.0 & -8.8 & 5 & - & 0.1 \\
\hline 5 & - & -1.0 & $\underline{\overline{6}}$ & 5.7 & 8.4 & $2 \overline{4} 0$ & 27.8 & 34.6 \\
\hline 6 & 4.5 & 5.9 & $\overline{7}$ & 3.6 & 1.2 & 1 & 9.0 & 11.4 \\
\hline 7 & 3.5 & 2.9 & $\overline{8}$ & 5.3 & 5.5 & 2 & - & $-\quad 0.4$ \\
\hline $\bar{I}$ & 6.1 & 5.1 & $\overline{9}$ & 4.9 & 5.2 & 3 & 17.6 & -20.3 \\
\hline$\underline{\underline{2}}$ & 8.3 & 6.6 & & & & 4 & 16.2 & -25.0 \\
\hline$\overline{\mathbf{3}}$ & 13.6 & 14.4 & 040 & 26.1 & 25.1 & 5 & - & 2.0 \\
\hline$\overline{4}$ & - & 1.3 & 1 & 6.2 & 4.0 & 6 & 3.4 & 0.7 \\
\hline$\overline{5}$ & 5.2 & 5.9 & 2 & 14.3 & 12.5 & $\overline{1}$ & 4.8 & 7.4 \\
\hline$\overline{6}$ & 18.3 & 21.5 & 3 & - & 1.2 & $\overline{2}$ & 7.7 & 10.5 \\
\hline$\overline{7}$ & 14.0 & 16.0 & 4 & 9.3 & 10.8 & $\overline{3}$ & 14.9 & -20.1 \\
\hline$\overline{8}$ & 11.0 & 10.1 & 5 & 12.0 & 10.6 & $\frac{0}{4}$ & - & 1.6 \\
\hline $1 \overline{3} 0$ & 12.0 & 16.3 & 6 & 10.7 & 8.6 & $\frac{4}{5}$ & 3.4 & $\begin{array}{l}-2.3 \\
-\end{array}$ \\
\hline 1 & 4.2 & 5.1 & 7 & 13.3 & 15.3 & $\frac{\pi}{6}$ & 9.9 & 10.8 \\
\hline 2 & 5.8 & 9.8 & $\underline{1}$ & 13.3 & 9.2 & $\frac{\square}{7}$ & 7.7 & 9.7 \\
\hline 3 & 24.1 & 23.7 & $\overline{2}$ & 5.0 & -3.7 & 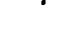 & & \\
\hline 4 & 26.4 & 23.2 & $\overline{\mathbf{3}}$ & 30.7 & -30.4 & & & \\
\hline 5 & 5.0 & 3.9 & $\underline{\overline{4}}$ & 9.5 & -8.7 & 050 & & \\
\hline 6 & - & 1.1 & $\overline{\overline{5}}$ & 11.0 & $\begin{array}{l}-9.9 \\
-\end{array}$ & 1 & 7.3 & 10.4 \\
\hline 7 & 5.9 & 8.4 & $\underline{6}$ & 11.6 & 12.5 & 2 & 12.5 & -13.8 \\
\hline 8 & - & -5.0 & $\overline{7}$ & 6.5 & 7.9 & 3 & 10.9 & -9.1 \\
\hline 9 & 4.1 & 4.5 & $\overline{8}$ & 11.2 & 7.4 & 4 & 15.1 & -11.8 \\
\hline$\overline{\mathbf{I}}$ & - & 2.0 & & & & $\underline{5}$ & - & -2.5 \\
\hline$\overline{2}$ & - & -2.5 & 140 & 18.6 & 20.7 & $\overline{1}$ & 16.6 & 16.5 \\
\hline$\overline{3}$ & - & 4.2 & 1 & 16.0 & 16.3 & $\underline{\overline{2}}$ & - & 6.0 \\
\hline$\overline{4}$ & & 1.0 & 2 & 7.7 & -8.5 & $\overline{\mathbf{2}}$ & - & 4.6 \\
\hline$\overline{\mathbf{5}}$ & - & 2.0 & 3 & 6.6 & -10.1 & $\underline{\mathbf{3}}$ & - & 2.8 \\
\hline$\overline{6}$ & 19.5 & 20.1 & 4 & 9.6 & -10.1 & $\overline{5}$ & 8.4 & 3.8 \\
\hline$\overline{7}$ & 13.7 & 12.6 & 5 & 6.2 & 7.1 & $\overline{6}$ & 9.0 & 11.2 \\
\hline \multirow[t]{2}{*}{$\overline{8}$} & 9.3 & 9.8 & $\overline{1}$ & 27.3 & 22.9 & $\overline{7}$ & 15.8 & 13.7 \\
\hline & & & $\overline{\overline{2}}$ & - & -4.2 & $\overline{8}$ & 5.7 & 5.8 \\
\hline 230 & 10.0 & 8.3 & $\overline{3}$ & - & -4.2 & & & \\
\hline 1 & 4.3 & 4.1 & $\underline{\overline{4}}$ & 7.5 & -8.7 & $1 \overrightarrow{5} 0$ & 20.5 & 22.0 \\
\hline 2 & 15.0 & -11.3 & $\overline{5}$ & - & -5.3 & 1 & 15.9 & 19.0 \\
\hline 3 & 10.6 & 6.7 & $\overline{6}$ & 4.9 & 5.4 & 2 & - & -5.6 \\
\hline 4 & 10.0 & 10.1 & $\overline{7}$ & 6.0 & 8.1 & 3 & 5.3 & -4.3 \\
\hline 5 & 13.1 & 11.2 & $\overline{8}$ & 9.8 & 7.4 & 4 & 10.7 & -8.0 \\
\hline 6 & 10.9 & 6.1 & $1 \overline{4} 0$ & 24.0 & 26.3 & 5 & - & -2.0 \\
\hline$\underline{1}$ & 5.3 & 3.8 & 1 & 7.5 & 8.3 & 6 & 4.0 & 4.2 \\
\hline$\underline{\overline{2}}$ & - & 0.9 & 2 & 5.3 & 7.0 & $\overline{\mathbf{I}}$ & 12.3 & 16.8 \\
\hline$\overline{\mathbf{3}}$ & 27.9 & 35.4 & 3 & 13.8 & -15.1 & $\underline{\underline{2}}$ & 3.9 & -4.5 \\
\hline$\underline{\overline{4}}$ & 16.0 & 17.5 & 4 & - & -2.5 & $\overline{3}$ & - & 2.2 \\
\hline$\overline{\mathbf{5}}$ & 10.3 & 13.5 & 5 & - & -2.3 & $\overline{\underline{4}}$ & - & 2.8 \\
\hline$\underline{\overline{6}}$ & 4.8 & 8.1 & 6 & 17.1 & 20.0 & $\overline{\mathbf{5}}$ & - & 5.7 \\
\hline$\overline{\overline{7}}$ & 3.9 & 2.7 & 7 & 6.8 & 9.2 & $\underline{\overline{6}}$ & - & 1.0 \\
\hline$\overline{8}$ & 6.8 & 6.9 & $\overline{1}$ & - & 6.8 & $\overline{7}$ & 7.4 & 8.5 \\
\hline
\end{tabular}


DINUCLEAR COBALT COMPLEXES

\begin{tabular}{|c|c|c|c|c|c|c|c|c|}
\hline$h k l$ & $F_{\mathrm{o}}$ & $F_{\mathrm{c}}$ & $h k l$ & $F_{\mathrm{o}}$ & $F_{\mathrm{c}}$ & $h k l$ & $F_{\mathrm{o}}$ & $F_{\mathrm{c}}$ \\
\hline $2 \overline{5} 0$ & 4.3 & 5.2 & 2 & 5.1 & 5.4 & $1 \overline{\overline{7}} 0$ & 12.0 & 11.0 \\
\hline 1 & - & 2.0 & 3 & 7.1 & 7.1 & 1 & 3.5 & 2.9 \\
\hline 2 & $\overline{-}$ & 0.0 & $1 \overline{6} 0$ & 3.2 & & 2 & 5.4 & 5.3 \\
\hline 3 & 24.5 & -27.5 & & & $\begin{array}{l}5.0 \\
3.3\end{array}$ & $\underline{3}$ & 3,5 & 4.9 \\
\hline 3 & 24.5 & -27.5 & 1 & 3.3 & 3.5 & $\overline{1}$ & - & 0.2 \\
\hline 4 & 14.9 & -13.0 & 2 & 4.2 & 7.2 & $\underline{\overline{2}}$ & 4.6 & 2.9 \\
\hline 5 & 5.1 & -4.9 & 3 & & 7.1 & $\overline{\overline{3}}$ & 11.5 & 9.0 \\
\hline 6 & 9.4 & 9.8 & $2 \overline{6} 0$ & - & 3.5 & & & \\
\hline 7 & 1.9 & 1.4 & 1 & 9.1 & 8.9 & & & \\
\hline$\overline{1}$ & 3.4 & -1.7 & 2 & - & 2.2 & $2 \overline{7} 0$ & 13.6 & 12.8 \\
\hline$\overline{2}$ & 6.0 & -5.6 & 3 & - & 0.8 & 1 & 4.8 & 6.7 \\
\hline$\overline{3}$ & - & -4.8 & 4 & 8.2 & -5.8 & 2 & 4.3 & -1.2 \\
\hline$\overline{4}$ & 8.5 & -12.3 & $\overline{1}$ & 6.4 & 11.3 & 3 & 3.9 & \\
\hline$\overline{5}$ & 6.5 & 7.8 & $\overline{2}$ & 6.4 & -12.4 & $\underline{4}$ & 10.0 & -9.3 \\
\hline$\overline{0}$ & 8.2 & 6.6 & $\overline{3}$ & 6.1 & -7.0 & $\overline{1}$ & 3.0 & 4.9 \\
\hline$\overline{7}$ & 16.6 & 16.5 & $\overline{4}$ & 8.5 & -10.5 & $\overline{\overline{2}}$ & 2.7 & 5.2 \\
\hline & & & 070 & 13.8 & 10.6 & & & \\
\hline 60 & 23.2 & 19.8 & 1 & - & -3.6 & & & \\
\hline & - & 4.6 & 2 & 8.5 & -3.5 & & & \\
\hline
\end{tabular}

The large complex ions are surrounded by eight chloride ions and eight water molecules, each of which form a distorted cube. Each chloride ion or water molecule is then coordinated to two complex ions. An idealized model of the structure can be seen in Figs. 3 and 4. From these figures it is clear that the structure can be described as consisting of sheets of chloride ions and water molecules interleaved by the complex ions. The sheets are bonded together in pairs by the complex ions, but there are only very weak bonds between two sheets not interleaved by the complex groups. In the sheets and between sheets and complex groups there are, in addition to ionic bonds also moderately strong hydrogen bonds; Fig. 3 .

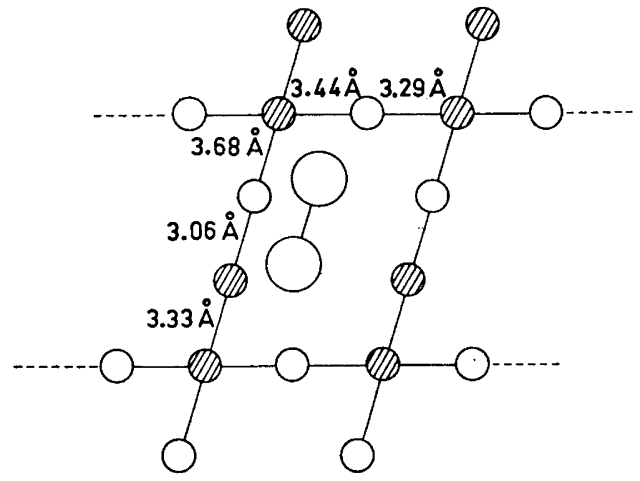

Fig. 3. A somewhat idealized model of the coordination around the complex ions, projected on (010); shaded, $\mathrm{H}_{2} \mathrm{O}$; white, $\mathrm{Cl}^{-}$.

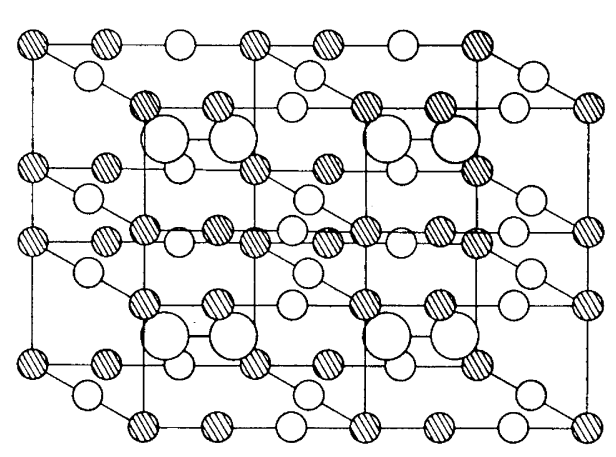

Fig. 4. An idealized model of the $\left[\left(\mathrm{NH}_{3}\right)_{4} \mathrm{Co}(\mathrm{OH})_{2} \mathrm{Co}\left(\mathrm{NH}_{3}\right)_{4}\right] \mathrm{Cl}_{4} \cdot 4 \mathrm{H}_{2} \mathrm{O}$ structure; shaded, $\mathrm{H}_{2} \mathrm{O}$; white, $\mathrm{Cl}^{-}$. 
The geometry of the complex ion $\left[\left(\mathrm{NH}_{3}\right)_{4} \mathrm{Co}(\mathrm{OH})_{2} \mathrm{Co}\left(\mathrm{NH}_{3}\right)_{4}\right]^{4+}$ may best be described as two octahedrons sharing one edge; Fig. 5. The distances between the central atoms and the ammonia ligands are normal. It is interesting that the bonds between the bridging hydroxyl groups and the cobalt atoms in this compound are shorter than the bonds between a bridging amide group and cobalt atoms in the compound $\left[\left(\mathrm{NH}_{3}\right)_{5} \mathrm{CoNH} \mathrm{No}_{2} \mathrm{Co}\left(\mathrm{NH}_{3}\right)_{5}\right]\left(\mathrm{NO}_{3}\right)_{5}$.

The two heights of the octahedrons can be expected to be nearly parallel, which means that the cobalt atoms, the bridging hydroxyl groups and four of the ammonia molecules should be in the same plane. The coordinates in the oblique axes system are transformed to an orthonormal system by the matrix ${ }^{18}$

$\left[\begin{array}{ccc}\sqrt{g_{11}} & \frac{g_{12}}{g_{11}} & \sqrt{g_{11}} \\ 0 & \frac{\sqrt{g^{33}}}{g_{11}} & \frac{\sqrt{g} \cdot g^{32}}{\sqrt{g_{11} \cdot g^{33}}} \\ 0 & 0 & \frac{1}{\sqrt{g^{33}}}\end{array}\right] \equiv\left[\begin{array}{ccc}6.66 & -2.231 & -2.897 \\ 0 & 7.49 & -1.212 \\ 0 & 0 & 9.21\end{array}\right]$

The $X$-axis in the new system is parallel to the $x$-axis in the oblique system. The $Y$-axis is perpendicular to $x$ and in the same plane as $x$ and $y$, while the $Z$-axis is perpendicular to this plane. The systems possess a common origin. In this axial system the equation of the plane through the cobalt atoms and the hydroxyl groups is

$$
-0.198 X+1.762 Y+0.132 Z=0
$$

The ammonia molecules 1 and 2 lie by only $0.04 \AA$ resp. $0.06 \AA$ units out of this plane, which means that within the limits of error the cobalt atoms, the hydroxyl groups and the ammonia molecules are coplanar. The angles between this plane and the ammonia-cobalt bonds which are not situated in

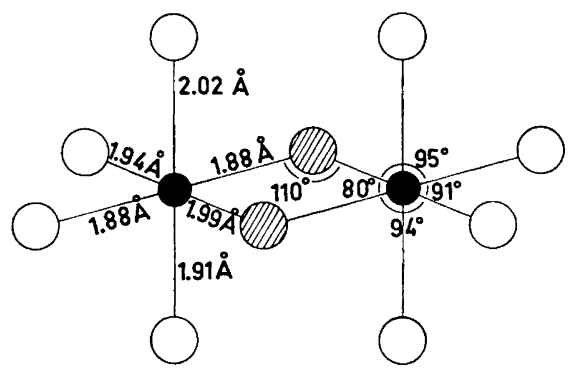

Fig. 5. The configuration of the ion $\left[\left(\mathrm{NH}_{3}\right)_{4} \mathrm{Co}(\mathrm{OH})_{2} \mathrm{Co}\left(\mathrm{NH}_{3}\right)_{4}\right]^{4}+$; shaded, $\mathrm{OH}^{-}$; white, $\mathrm{NH}_{3}$; black, Co. 
Table 3. Interatomic distances in $\left[\left(\mathrm{NH}_{3}\right)_{4} \mathrm{Co}(\mathrm{OH})_{2} \mathrm{Co}\left(\mathrm{NH}_{3}\right)_{4}\right] \mathrm{Cl}_{4} \cdot 4 \mathrm{H}_{2} \mathrm{O}$.

$\begin{array}{ll}\mathrm{Co} & -\mathrm{Co} \\ \mathrm{Co} & -\mathrm{N}_{1} \\ \mathrm{Co} & -\mathrm{N}_{2} \\ \mathrm{Co} & -\mathrm{N}_{3} \\ \mathrm{Co} & -\mathrm{N}_{4} \\ \mathrm{Co} & -\mathrm{N}^{\prime}(\text { mean }) \\ \mathrm{Co} & -\mathrm{O}_{1} \\ \mathrm{Co} & -\mathrm{O}_{1}^{\prime} \\ \mathrm{Co} & -\mathrm{O}^{\prime}(\text { mean }) \\ \mathrm{O} & -\mathrm{O}_{1}^{\prime} \\ \mathrm{Cl} & -\mathrm{O}_{2}\left(\mathrm{H}_{2} \mathrm{O}\right) \\ \mathrm{Cl} & -\mathrm{O}_{3}\left(\mathrm{H}_{2} \mathrm{O}\right) \\ \mathrm{Cl} & -\mathrm{O}_{2}^{\prime}\left(\mathrm{H}_{2} \mathrm{O}\right) \\ \mathrm{Cl} & -\mathrm{O}_{3}{ }^{\prime}\left(\mathrm{H}_{2} \mathrm{O}\right) \\ \mathrm{Cl} & -\mathrm{O}_{3}{ }^{\prime} \\ \mathrm{O}_{2}\left(\mathrm{H}_{2} \mathrm{O}\right)-\mathrm{O}_{3}\left(\mathrm{H}_{2} \mathrm{O}\right) \\ \mathrm{N}_{3} & -\mathrm{Cl}_{1} \\ \mathrm{~N}_{4} & -\mathrm{Cl}_{1} \\ \mathrm{~N}_{3} & -\mathrm{O}_{2}\left(\mathrm{H}_{2} \mathrm{O}\right) \\ \mathrm{N}_{4} & -\mathrm{O}_{3}\left(\mathrm{H}_{2} \mathrm{O}\right) \\ \mathrm{O}^{3} & -\mathrm{Cl}_{2}\end{array}$

$\begin{array}{ll}2.97 & \pm 0.01 \AA \\ 2.02 & \pm 0.06 \\ 1.91 & \pm 0.06 \\ 1.88 & \pm 0.07 \\ 1.94 & \pm 0.06 \\ 1.94 & \pm 0.06 \\ 1.99 & \pm 0.06 \\ 1.88 & \pm 0.08 \\ 1.94 & \pm 0.07 \\ 2.49 & \pm 0.06 \\ 3.41 & \pm 0.07 \\ 3.22 & \pm 0.06 \\ 3.44 & \pm 0.08 \\ 3.29 & \pm 0.06 \\ 3.07 & \pm 0.06 \\ 3.33 & \pm 0.06 \\ 3.30 & \pm 0.06 \\ 3.28 & \end{array}$

this plane should be $90^{\circ}$. The measured values are $87^{\circ}$ and $85^{\circ}$. The remaining bond angles in the complex ion can be seen in Fig. 5. The angles $\mathrm{O}-\mathrm{Co}-\mathrm{O}$, equal to $80^{\circ}$, and the angles $\mathrm{Co}-\mathrm{O}-\mathrm{Co}$, equal to $100^{\circ}$, are especially interesting. If pure covalent bonds are assumed, the first bond angle should be $90^{\circ}$, while the second should be $120^{\circ}$ or $109^{\circ}$, depending on whether the oxygen atom adopts $\mathrm{sp}^{2}$ or $\mathrm{sp}^{3}$ hydridisation. In the present case the sum of the two pairs of bond angles cannot be greater than $360^{\circ}$. As the four atoms are coplanar, the $\mathrm{O}-\mathrm{Co}-\mathrm{O}$ angle can be expected to be about $80^{\circ}$, while the $\mathrm{Co}-\mathrm{O}-\mathrm{Co}$ angle should be about $100^{\circ}$. A similar result can also be anticipated if ionic bonds are assumed. The higher charge on the cobalt atoms demands the Co-Co distance to be as great as possible. Equilibrium is first attained when there is an appreciable overlap between the outer orbitals of the oxygen atoms. The repulsion due to the ionic charge on the hydroxyl groups can be neglected in comparison with the Born repulsion.

No attempt has been made to find the hydrogen atoms from the Fourier synthesis. In those cases where hydrogen bonds are formed it is possible to locate the hydrogen atoms approximately. It is then interesting to note that

$\begin{array}{ll}\text { Table 4. Bond angles in }\left[\left(\mathrm{NH}_{3}\right)_{4} \mathrm{Co}(\mathrm{OH})_{2} \mathrm{Co}\left(\mathrm{NH}_{3}\right)_{4}\right] \mathrm{Cl}_{4} \cdot 4 \mathrm{H}_{2} \mathrm{O} \\ \mathrm{N}_{3}-\mathrm{Co}-\mathrm{N}_{4} & 91^{\circ} \\ \mathrm{N}_{3}-\mathrm{Co}-\mathrm{O}_{1} & 95^{\circ} \\ \mathrm{N}_{4}^{\prime}-\mathrm{Co}-\mathrm{O}_{1}^{\prime} & 94^{\circ} \\ \mathrm{O}^{\prime}-\mathrm{Co}-\mathrm{O}_{1} & 80^{\circ} \\ \mathrm{Co}-\mathrm{O}_{1}-\mathrm{Co}^{\prime} & 110^{\circ} \\ \mathrm{N}_{1}-\mathrm{Co}\left(\mathrm{Plane}^{\circ} \mathrm{Co}_{1} \mathrm{O}_{1} \mathrm{O}_{1}^{\prime}\right) & 87^{\circ} \\ \mathrm{N}_{2}-\mathrm{Co}\left(\mathrm{Plane}_{\mathrm{CoO}} \mathrm{CoO}_{1} \mathrm{O}_{1}^{\prime}\right) & 85^{\circ} \\ \mathrm{Co}-\mathrm{O}_{1} \ldots \mathrm{Cl}_{1} & 105^{\circ}\end{array}$

The standard deviation is $\pm 6^{\circ}$.

Acta Chem. Scand. 17 (1963) No. 1 
the hydroxylic hydrogen atoms are not situated in the hydroxyl-cobalt plane. Thus if the complex bonds are regarded from the point of view of the valency bond theory, it would indicate $\mathrm{sp}^{3}$ hybridisation on the oxygen atoms. Water in other complex compounds seems to be coordinated in such a way that its twofold axis and the metal-oxygen axis are colinear.

Acknowledgements. The author wishes to thank professor C. Brosset for his kind interest. Thanks are also due to Mrs. M. Biéth and Mr. S. Toll for technical assistance.

\section{REFERENCES}

1. Gentele, J. G. J. prakt. Chem. 67 (1856) 137.

2. Werner, A. Ber. 40 (1907) 4434.

3. Werner, A. Ber. 40 (1907) 4817.

4. Werner, A. Ber. 01 (1908) 3884.

5. Werner, A. Ann. 386 (1912) 16.

6. Dubsky, J. V. J. prakt. Chem. (2) 90 (1914) 82.

7. Frank, H. Wissensch. Ind. 2 (1923) 8.

8. Rosenbohm, E. Z. physik. Chem. 93 (1919) 693.

9. Jackson, L. C. Phil. Mag. (7) 2 (1926) 87.

10. Jackson, L. C. Phil. Mag. 4 (1927) 1070.

11. Shibata, Y. J. Coll. Sci. Imp. Univ. Tokyo 37 (1916) 19.

12. Inamura, Y. and Kondo, Y. J. Chem. Soc. Japan Pure Chem. Sect. 74 (1953) 627.

13. Ve'mura, T. and Sue'da, H. Bull. Chem. Soc. Japan 10 (1935) 50.

14. Skanavi-Grigor'eva, M. S. and Staroverova, I. P. Zhur. Obshchei Khim. 27 (1957) 573.

15. Westman, S., Blomqvist, G. and Åsbrink, S. Arkiv Kemi 14 (1959) 535.

16. Åsbrink, S., Blomqvist, G. and Westman, S. Arkiv Kemi 14 (1959) 545.

17. Cruickshank, D. W. J. Acta Cryst. 2 (1949) 65.

18. International Tables for X-Ray Crystallography, Vol. II, The Kynoch Press 1959, p. 60 .

Received June 21, 1962. 